\section{Interval Fuzzy Modeling Applied to Wiener Models With Uncertainties}

Igor Škrjanc, Sašo Blažič, and Osvaldo Enrique Agamennoni

\begin{abstract}
This correspondence addresses the problem of interval fuzzy model identification and its use in the case of the robust Wiener model. The method combines a fuzzy identification methodology with some ideas from linear programming theory. On a finite set of measured data, an optimality criterion which minimizes the maximum estimation error between the data and the proposed fuzzy model output is used. The min-max optimization problem can then be seen as a linear programming problem that is solved to estimate the parameters of the fuzzy model in each fuzzy domain. This results in lower and upper fuzzy models that define the confidence interval of the observed data. The model is called the interval fuzzy model and is used to approximate the static nonlinearity in the case of the Wiener model with uncertainties. The resulting model has the potential to be used in the areas of robust control and fault detection.
\end{abstract}

Index Terms - Interval fuzzy model, linear programming, min-max optimization, Wiener model.

\section{INTRODUCTION}

Interval modeling is a methodology to approximate function families that are given as a finite set of input and output measurements. In connection with fuzzy models, it can be successfully applied to approximate nonlinear function families. The result of such an application is the interval of parameters that define the confidence band containing the whole measurement set.

This type of modeling in combination with other classical modeling methods enables the investigation of uncertainty problems in nonlinear dynamical systems. Fuzzy linear regression, proposed in [1], is still one of the most widely used approaches in fuzzy modeling due to its use of linear programming and because of its simplicity. This approach has influenced the investigation of the uncertainties problem in recent years. In [2] the possibility fuzzy linear regression was used; this is based on an estimation of the reliability of the data defined by a reliability threshold value. A modification of this approach was reported in [3], where the bounds of the measurement interval are treated as fuzzy, and in [4], where a method is presented that can detect the outliers. Another approach associated with modeling systems with uncertainties is presented in [5]-[7]. These approaches are called type-2 fuzzy systems, and they translate all the uncertainties of the measurement into uncertainties about the membership functions. This approach results in a notation that gives the third dimension to the membership functions of the classical type. This dimension is called a possibility grade and provides the possibility for the classical or type-1 membership degree. In our approach the membership functions are certain and the uncertainty is modeled by the time-invariant interval parameters of the system. The estimation of the model parameters requires a linear programming method.

To demonstrate the possible use of the interval fuzzy model, it is implemented in the robust Wiener model, which consists of a dynamic

Manuscript received November 14, 2003; revised November 11, 2004. This work was supported by a bilateral project between Argentina and Slovenia under Grant Slo-Arg 2001-2004. This paper was recommended by Associate Editor Fernando Gomide.

I. Škrjanc and S. Blažič are with the Faculty of Electrical Engineering, University of Ljubljana, Slovenia, 1000 Ljubljana, Slovenia (e-mail: igor.skrjanc@fe.uni-lj.si).

O. E. Agamennoni is with the Universidad Nacional del Sur, Bahia Blanca, 8000, Argentina.

Digital Object Identifier 10.1109/TSMCB.2005.850166 linear block in cascade with static nonlinearity at the output. The implementation of the interval fuzzy model provides the possibility to model nonlinear dynamical systems with uncertainty or the families of nonlinear systems with uncertain physical parameters.

The interval fuzzy models can also be very efficiently used in the case of fault detection, where the model of the normal dataset or the normal functions with some normal uncertainties is identified and compared with the tested data. When the data of a tested function do not belong to the confidence interval, then we can assume the faulty functioning of the corresponding system.

The correspondence is organized as follows. In Section II the background to interval fuzzy modeling is provided, and in Section III the use of the interval fuzzy model is shown for the case of the robust Wiener model. A simple example is provided to illustrate the proposed procedure. At the end some conclusions are given.

\section{INTERVAL FUZZY MODEL IDENTIFICATION}

We assume a typical fuzzy model given in the form

$$
\begin{aligned}
\mathbf{R}_{j}: & \text { if } x_{p 1} \text { is } \mathbf{A}_{1, k_{1}} \text { and } \ldots \text { and } x_{p q} \text { is } \mathbf{A}_{q, k_{q}} \\
& \quad \operatorname{then} y=\theta_{j, 0}+\theta_{j, 1} x_{1}+\theta_{j, 2} x_{2}+\ldots+\theta_{j, n} x_{n} \\
& j=1, \ldots, m \\
& k_{1}=1, \ldots, f_{1} \quad k_{2}=1, \ldots, f_{2} \ldots \quad k_{q}=1, \ldots, f_{q} .
\end{aligned}
$$

The $q$-element vector $\mathbf{x}_{p}^{T}=\left[x_{p 1}, \ldots, x_{p q}\right]$ denotes the input or variables in premise, and the variable $y$ is the output of the model. With each variable in premise $x_{p i}(i=1, \ldots, q), f_{i}$ fuzzy sets $\left(\mathbf{A}_{i, 1}, \ldots, \mathbf{A}_{i, f_{i}}\right)$ are connected, and each fuzzy set $\mathbf{A}_{i, k_{i}}$ $\left(k_{i}=1, \ldots, f_{i}\right)$ is associated with a real-valued function $\mu_{A_{i, k_{i}}}\left(x_{p i}\right)$ : $\mathbb{R} \rightarrow[0,1]$, that produces the membership grade of the variable $x_{p i}$ with respect to the fuzzy set $\mathbf{A}_{i, k_{i}}$. To make the list of fuzzy rules complete, all possible variations of the fuzzy sets are given in (1), yielding the number of fuzzy rules $m=f_{1} \times f_{2} \times \cdots \times f_{q}$. Note that while the indices $k_{1}, \ldots, k_{q}$ change all the possible variations, $j$ runs from 1 to $m$. The variables $x_{p i}$ are not the only inputs to the fuzzy system. Implicitly, the $(n+1)$-element vector $\mathbf{x}^{T}=\left[1, x_{1}, \ldots, x_{n}\right]$ also represents an input to the system. It is usually referred to as the consequence vector.

The output of the fuzzy model defined in (1) is given in the matrix form as follows:

$$
y=\boldsymbol{\beta}^{T}\left(\mathbf{x}_{p}\right) \boldsymbol{\Theta} \mathbf{x}
$$

where the membership vector

$$
\boldsymbol{\beta}^{T}\left(\mathbf{x}_{p}\right)=\left[\beta_{1}\left(\mathbf{x}_{p}\right), \ldots, \beta_{m}\left(\mathbf{x}_{p}\right)\right]
$$

is composed of normalized degrees of fulfillment

$$
\begin{aligned}
& \beta_{j}\left(\mathbf{x}_{p}\right)=\frac{\mu_{A_{1, k_{1}}}\left(x_{p 1}\right) \ldots \mu_{A_{q}, k_{q}}\left(x_{p q}\right)}{\sum_{k_{1}=1}^{f_{1}} \cdots \sum_{k_{q}=1}^{f_{q}} \mu_{A_{1, k_{1}}}\left(x_{p 1}\right) \ldots \mu_{A_{q, k_{q}}}\left(x_{p q}\right)}, \\
& j=1, \ldots, m
\end{aligned}
$$

and the matrix of fuzzy model parameters

$$
\boldsymbol{\Theta}=\left[\begin{array}{c}
\boldsymbol{\theta}_{1}^{T} \\
\vdots \\
\boldsymbol{\theta}_{m}^{T}
\end{array}\right]
$$

is composed of vectors of parameters in individual fuzzy domains

$$
\boldsymbol{\theta}_{j}^{T}=\left[\theta_{j, 0}, \theta_{j, 1}, \ldots, \theta_{j, n}\right], \quad j=1, \ldots, m .
$$


It is obvious that $\sum_{j=1}^{m} \beta_{j}\left(\mathbf{x}_{p}\right)=1$ irrespective of $\mathbf{x}_{p}$ as long as the denominator of $\beta_{j}\left(\mathbf{x}_{p}\right)$ in (4) is not equal to zero (that can easily be prevented by stretching the membership functions over the whole potential area of $\mathbf{x}_{p}$ ).

From (2) it is evident that the output of a fuzzy system is a function of the premise vector $\mathbf{x}_{p}$ ( $q$-dimensional) and the consequence vector $\mathbf{x}[(n+1)$-dimensional]. The dimension of the input space $d$ may be lower than $(q+n+1)$ since it is very usual to have the same variables present in vectors $\mathbf{x}_{p}$ and $\mathbf{x}$

$$
\max (q, n+1) \leq d \leq q+n+1
$$

The fuzzy model in the form given in (2) is referred to as the affine Takagi-Sugeno model and can be used to approximate an arbitrary function that maps the compact set $\mathbf{C} \subset \mathbb{R}^{d}$ to $\mathbb{R}$ with any desired degree of accuracy [8]-[10]. The generality can be proven by the Stone-Weierstrass theorem [11], which indicates that any continuous function can be approximated by a fuzzy basis function expansion [12].

Let us consider a class of nonlinear functions $\mathcal{G}$

$$
\mathcal{G}=\{g: \mathbf{C} \rightarrow \mathbb{R}\}
$$

Our goal is to find the exact upper bound $\bar{g}$ and the exact lower bound $g$ of the functions in the class $\mathcal{G}$. The upper bound $\bar{g}$ satisfies the following two conditions for each $\mathbf{z} \in \mathbf{C}$ and arbitrary $\varepsilon>0$ :

$$
\begin{aligned}
& \bar{g}(\mathbf{z}) \geq \max _{g \in \mathcal{G}} g(\mathbf{z}) \\
& \exists g \in \mathcal{G}: \bar{g}(\mathbf{z})<g(\mathbf{z})+\varepsilon
\end{aligned}
$$

and the lower bound $\underline{g}$ satisfies

$$
\begin{aligned}
& \underline{g}(\mathbf{z}) \leq \max _{g \in \mathcal{G}} g(\mathbf{z}) \\
& \exists g \in \mathcal{G}: \underline{g}(\mathbf{z})>g(\mathbf{z})+\varepsilon .
\end{aligned}
$$

It is impossible to obtain the bounds defined in (9) and (10) since this would require infinite amount of data. In our case we deal with the finite set of measured output values $\mathbf{Y}=\left\{y_{1}, \ldots, y_{N}\right\}$ and the finite set of inputs $\mathbf{Z}=\left\{\mathbf{z}_{1}, \ldots, \mathbf{z}_{N}\right\}$

$$
y_{i}=g\left(\mathbf{z}_{i}\right), \quad g \in \mathcal{G}, \quad \mathbf{z}_{i} \in \mathbf{C} \subset \mathbb{R}^{d}, \quad y_{i} \in \mathbb{R}, \quad i=1, \ldots, N .
$$

Note that the data are obtained by sampling different functions from $\mathcal{G}$ with arbitrary values of $\mathbf{z}$.

The idea of robust interval fuzzy modeling is to find a lower fuzzy function $f$ and an upper fuzzy function $\bar{f}$ that approximate $g$ and $\bar{g}$, respectively. They have to satisfy the following condition:

$$
\underline{f}\left(\mathbf{z}_{i}\right) \leq y_{i} \leq \bar{f}\left(\mathbf{z}_{i}\right), \quad \forall \mathbf{z}_{i} \in \mathbf{Z} .
$$

The main request in defining the band is that it is as narrow as possible according to the proposed constraints. Our approach using the fuzzy function approximation can be viewed as a generalization of the piecewise linear approach [13] and gives a better approximation, or at least a much narrower approximation band.

The upper and lower fuzzy functions, respectively, can be found by solving the following optimization problems:

$$
\begin{aligned}
& \min _{\underline{f}} \max _{z_{i} \in \mathbf{Z}}\left|y_{i}-\underline{f}\left(\mathbf{z}_{i}\right)\right| \text { subject to } y_{i}-\underline{f}\left(\mathbf{z}_{i}\right) \geq 0 \\
& \min _{\bar{f}} \max _{z_{i} \in \mathbf{Z}}\left|y_{i}-\bar{f}\left(\mathbf{z}_{i}\right)\right| \text { subject to } y_{i}-\bar{f}\left(\mathbf{z}_{i}\right) \leq 0 .
\end{aligned}
$$

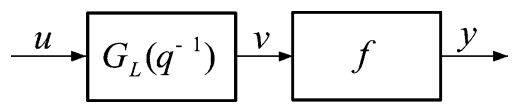

Fig. 1. Structure of the Wiener model.

The solutions to both problems can be found by linear programming, because both problems can be viewed as linear programming problems if fuzzy models (2) are used for $\underline{f}\left(\mathbf{z}_{i}\right)$ and $\bar{f}\left(\mathbf{z}_{i}\right)$

$$
\underline{f}\left(\mathbf{z}_{i}\right)=\boldsymbol{\beta}^{T}\left(\mathbf{x}_{p i}\right) \underline{\boldsymbol{\Theta}} \mathbf{x}_{i} \quad \bar{f}\left(\mathbf{z}_{i}\right)=\boldsymbol{\beta}^{T}\left(\mathbf{x}_{p i}\right) \overline{\boldsymbol{\Theta}} \mathbf{x}_{i} .
$$

The min-max optimization problems in (13) can be solved as the linear programming problems of minimizing $\lambda_{1}$ and $\lambda_{2}$, subject to the inequalities

$$
\begin{aligned}
& y_{i}-\sum_{j=1}^{m} \beta_{j}\left(\mathbf{x}_{p i}\right) \underline{\boldsymbol{\theta}}_{j}^{T} \mathbf{x}_{i} \leq \lambda_{1}, \quad i=1, \ldots, N \\
& y_{i}-\sum_{j=1}^{m} \beta_{j}\left(\mathbf{x}_{p i}\right) \overline{\boldsymbol{\theta}}_{j}^{T} \mathbf{x}_{i} \geq 0, \quad i=1, \ldots, N, \lambda_{1} \geq 0
\end{aligned}
$$

and

$$
\begin{aligned}
-y_{i}+\sum_{j=1}^{m} \beta_{j}\left(\mathbf{x}_{p i}\right) \overline{\boldsymbol{\theta}}_{j}^{T} \mathbf{x}_{i} \leq \lambda_{2}, \quad i=1, \ldots, N \\
y_{i}-\sum_{j=1}^{m} \beta_{j}\left(\mathbf{x}_{p i}\right) \underline{\boldsymbol{\theta}}_{j}^{T} \mathbf{x}_{i} \leq 0, \quad i=1, \ldots, N, \lambda_{2} \geq 0
\end{aligned}
$$

on the parameters $\underline{\boldsymbol{\theta}}_{j}, \overline{\boldsymbol{\theta}}_{j}, j=1, \ldots, m$, and $\lambda_{1}$ and $\lambda_{2}$ that stand for the maximum approximation errors of both approximation functions.

Due to the numerical problems, the above-mentioned algorithm becomes a hard optimization problem that requires an enormous amount of time, and so the solution $\underline{\boldsymbol{\theta}}_{j}, \overline{\boldsymbol{\theta}}_{j}, j=1, \ldots, m$ cannot be obtained in a reasonable time. This problem occurs in the case of a larger number of fuzzy parameters. To overcome this problem, the initial parameters of the interval fuzzy model should be chosen to be close to the optimal ones. The latter are obtained by using the ordinary fuzzy least squares methods.

\section{INTERVAl FuZzy Model USED IN THE WIENER MODEL}

Fading memory systems, e.g., Wiener-like models, enable a simple presentation of nonlinear systems. A Wiener model consists of a dynamic linear block with a normalized transfer function gain in cascade with a static nonlinearity at the output as it is shown in Fig. 1 with $v$ as the intermediate variable at the output of the linear block with the transfer function $G_{L}\left(q^{-1}\right)$, and $f$ as the static nonlinearity.

Many descriptions of static nonlinearity are possible: piecewise linear functions [13], an inverse polynomial, a polynomial spline, a fuzzy static model, etc. All these methods enable the approximation of different nonlinear static functions and the invertibility which is necessary to identify the Wiener model.

Different approaches of Wiener-model identification are found in the literature. The most frequently used is the nonlinear-linear (N-L) approach, which is the most comprehensible and ensures an accurate description of the static nonlinearity [14]. This approach requires steady-state data. The main disadvantage of this method is that it does not guarantee an unbiased estimation in an uncertain, noisy environment.

Another approach to modeling of Wiener-type systems is proposed in the present correspondence. The system under consideration has the structure as depicted in Fig. 1, but is uncertain in the parameters. Moreover, its output is corrupted with the limited measurement noise or disturbance. The proposed INFUMO model is used to model such systems, i.e., the upper and the lower bound of the system response can be found for arbitrary excitation signal. 

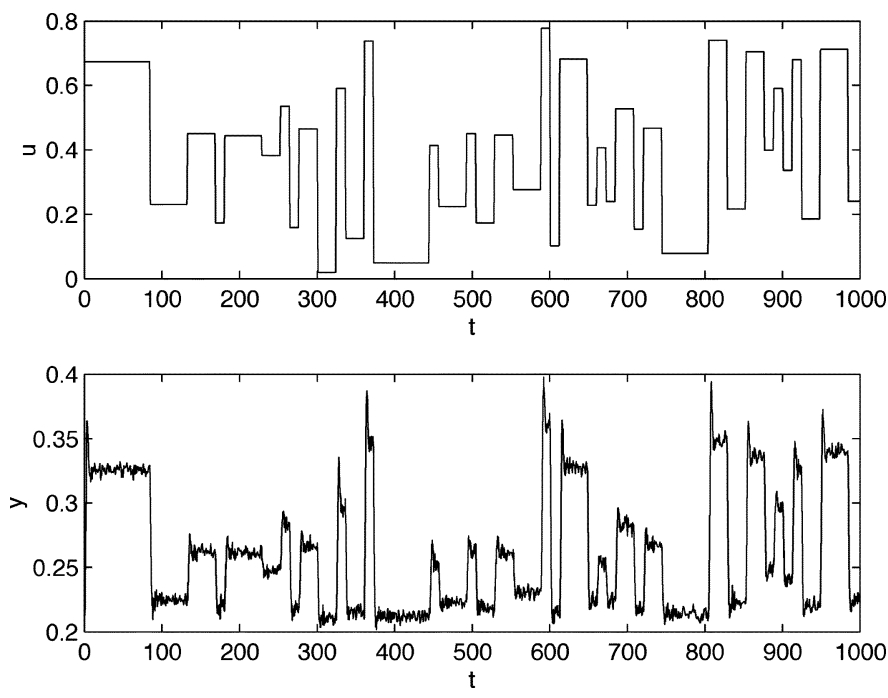

Fig. 2. Identification data.

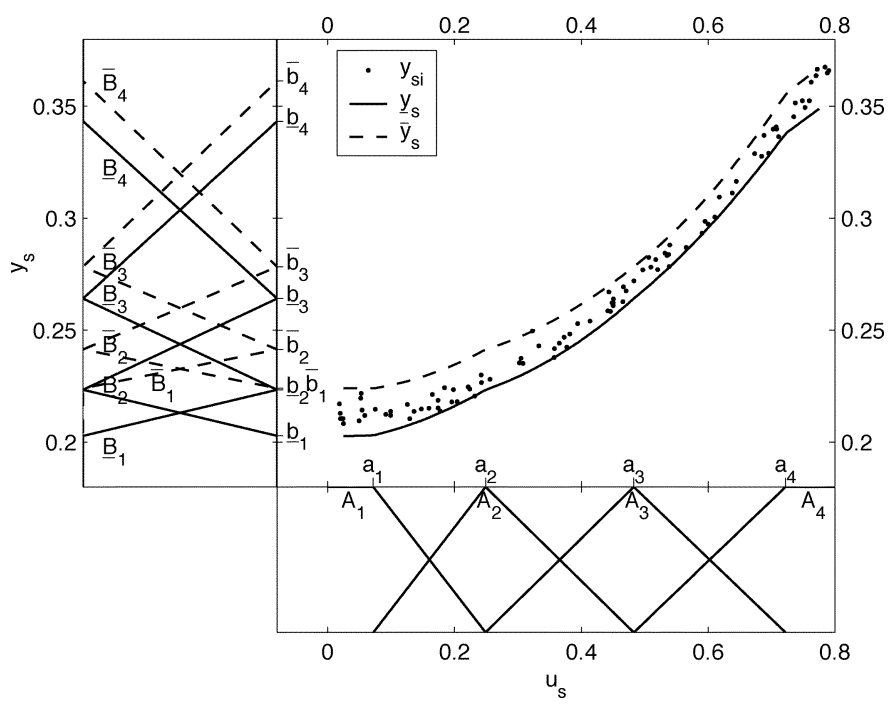

Fig. 3. Nonlinear static curve and membership functions.

A simulated experiment was conceived to illustrate the proposed approach. The excitation signal had to be designed to obtain the information about the steady-state behavior of the system. There is the measurement noise of limited amplitude present at the output of the system. The input and output signals are shown in Fig. 2. The steady-state curve of the simulated system is shown in Fig. 3, where the points correspond to the data pairs $\left(u_{s i}, y_{s i}\right)$ of the input variable $u$ and the corresponding output variable $y$ during steady-state. The dataset of steady-state points is a nonequidistant set of data, and it is spread around the nominal static curve because of the noisy output variable. This set of steady-state points is now modeled by the interval fuzzy model to obtain the lower and upper bounds of the whole set. When the set of steady-state points is rich enough, in the sense that the whole operating region is sufficiently covered by the data pairs $\left(u_{s i}, y_{s i}\right)$, then by using interval fuzzy modeling we can assume that the nominal static curve is always between the upper and lower bounds. These bounds are obtained by identifying the following interval fuzzy model:

$$
\begin{aligned}
& \overline{\mathbf{R}}_{j}: \text { if } u_{s} \text { is } \mathbf{A}_{j} \text { then } \bar{y}_{s}=\bar{\theta}_{j, 1} u_{s}+\bar{\theta}_{j, 0} \\
& \underline{\mathbf{R}}_{j}: \text { if } u_{s} \text { is } \mathbf{A}_{j} \text { then } \underline{y}_{s}=\underline{\theta}_{j, 1} u_{s}+\underline{\theta}_{j, 0}, \quad j=1, \ldots, 4
\end{aligned}
$$

with $y_{s}$ as the output variable in the steady-state and $u_{s}$ the corresponding input variable. We chose four fuzzy domains to model the system. The parameters of the interval fuzzy model are obtained solving the linear programming problem stated in (15) and (16) where the variables $\mathbf{x}_{p}$ and $\mathbf{x}$ are substituted by $u_{s}$ and $\left[1, u_{s}\right]^{T}$, respectively, and $m=4$. This problem was solved numerically using initial values of parameters that are obtained by the least squares method. The optimization produced the following results:

$$
\begin{aligned}
& \overline{\boldsymbol{\theta}}_{1}^{T}=[0.2240,0.0003] \quad \underline{\boldsymbol{\theta}}_{1}^{T}=[0.2024,0.0089] \\
& \overline{\boldsymbol{\theta}}_{2}^{T}=[0.2276,0.0550] \quad \underline{\boldsymbol{\theta}}_{2}^{T}=[0.2092,0.0571] \\
& \overline{\boldsymbol{\theta}}_{3}^{T}=[0.2191,0.1226] \\
& \overline{\boldsymbol{\theta}}_{3}^{T}=[0.1993,0.1342] \\
& \overline{\boldsymbol{\theta}}_{4}^{T}=[0.1928,0.2251] \quad \underline{\boldsymbol{\theta}}_{4}^{T}=[0.1866,0.2093] .
\end{aligned}
$$

The distributions of the membership functions are shown in Fig. 3, which also depicts the upper and lower bounds. It should be noted that the positioning of the membership functions is not treated in this correspondence, although this is well known to be a very important topic. Any existing method of positioning can be applied and combined with our approach. In our case, the c-means clustering algorithm was used [15], [16] to obtain the vertices of four membership functions $A_{j}$ $(j=1, \ldots, 4)$. These membership functions are shown in the bottom part of Fig. 3. Thus, the static nonlinear mapping in Fig. 1 has been identified.

The linear dynamic block $G_{L}\left(q^{-1}\right)$ still has to be identified. It has the signal $u$ at the input and $v$ at the output (see Fig. 1). Since the model (17) was obtained in the steady-state and the gain of $G_{L}\left(q^{-1}\right)$ is unity, the static mapping from $v$ to $y$ (marked with $f$ in Fig. 1) is equivalent to the static mapping from $u_{s}$ to $y_{s}$ that is described by the INFUMO model (17). To calculate the intermediate variable $v$ from $y$ the inverse static nonlinearity is required. In order to achieve this, the membership functions $A_{j}$ have to be transformed by the upper and the lower fuzzy model to obtain membership functions $\bar{B}_{j}$ and $\underline{B}_{j}$, respectively (see Fig. 3). The membership functions $\mu_{A_{j}}(j=1, \ldots, 4)$ are mapped to the output domain across the fuzzy functions (14) to obtain the membership functions $\mu_{\bar{B}_{j}}$ and $\mu_{\underline{B}}$, as shown in Fig. 3. The consequent part of the rules of the static mapping $y-v$ are obtained by solving the linear programming problem stated in (15) and (16)

$$
\begin{array}{r}
\overline{\mathbf{R}}_{j}^{i n v}: \text { if } y(k) \text { is } \overline{\mathbf{B}}_{j} \text { then } \bar{v}(k)=\bar{\theta}_{j, 1}^{i n v} y(k)+\bar{\theta}_{j, 0}^{i n v} \\
\underline{\mathbf{R}}_{j}^{i n v}: \text { if } y(k) \text { is } \underline{\mathbf{B}}_{j} \text { then } \underline{v}(k)=\underline{\theta}_{j, 1}^{i n v} y(k)+\underline{\theta}_{j, 0}^{i n v} \\
j=1, \ldots, 4 .
\end{array}
$$

The output variable $y$ is mapped by the inverse static nonlinearity defined in (19) to obtain the upper and lower intermediate variables $\bar{v}$ and $\underline{v}$, respectively. The average intermediate variable is then formed as $\tilde{v}=(\bar{v}+\underline{v}) / 2$. The transfer function $G_{L}\left(q^{-1}\right)$ formed as the relation between the input variable $u$ and the intermediate variable $v$ is estimated using the ARX identification method [17] on the data pairs $(u(k), \tilde{v}(k))$. The following linear transfer function is obtained

$$
G_{L}\left(q^{-1}\right)=\frac{0.3405 q^{-1}+0.2430 q^{-2}}{1-0.7853 q^{-1}+0.3674 q^{-2}}
$$

with the sampling time $T_{s}=1 \mathrm{~s}$. Note that the gain of this transfer function is not exactly 1 , since all the parameters of the transfer function are identified independently. Nevertheless, the gain is very close to 1 . 


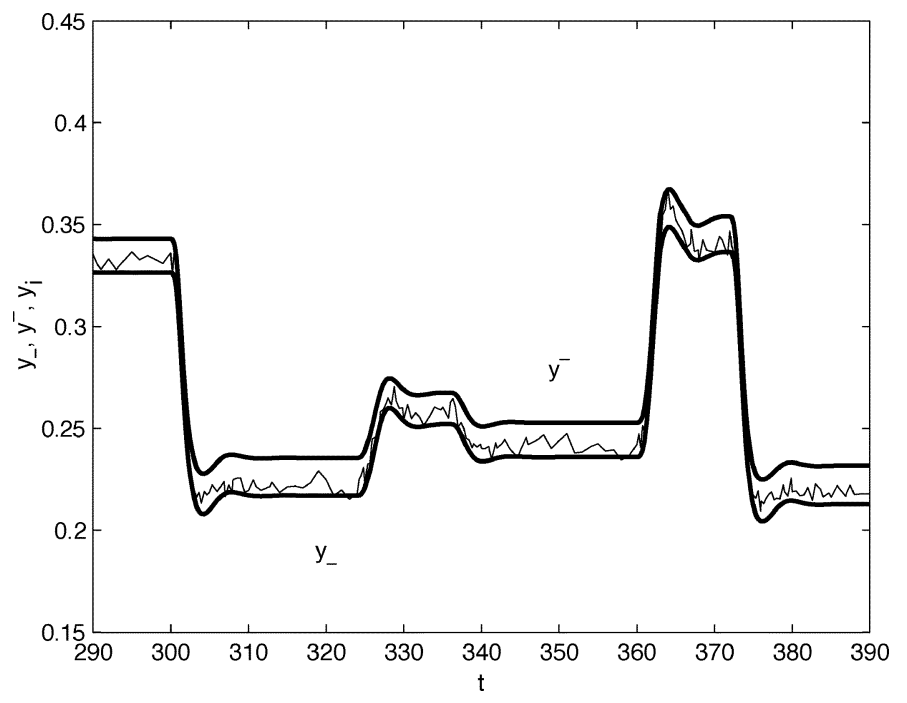

Fig. 4. Lower and upper bounds of the robust Wiener model.

The resulting robust Wiener model is now defined combining the linear part $[(20)]$ in the form of the difference equation and the static nonlinearity in fuzzy form [(14)] as follows:

$$
\begin{aligned}
v(k)= & 0.7853 v(k-1)-0.3674 v(k-2) \\
& +0.3405 u(k-1)+0.2430 u(k-2) \\
\bar{y}(k)= & \boldsymbol{\beta}^{T}(v(k)) \overline{\boldsymbol{\Theta}} \mathbf{x}(k) \\
\underline{y}(k)= & \boldsymbol{\beta}^{T}(v(k)) \underline{\boldsymbol{\Theta}} \mathbf{x}(k)
\end{aligned}
$$

with

$$
\begin{gathered}
\mathbf{x}(k)=\left[\begin{array}{c}
1 \\
v(k)
\end{array}\right] \\
\overline{\boldsymbol{\Theta}}=\left[\begin{array}{c}
\overline{\boldsymbol{\theta}}_{1}^{T} \\
\overline{\boldsymbol{\theta}}_{2}^{T} \\
\overline{\boldsymbol{\theta}}_{3}^{T} \\
\overline{\boldsymbol{\theta}}_{4}^{T}
\end{array}\right] \\
\underline{\boldsymbol{\Theta}}=\left[\begin{array}{c}
\underline{\boldsymbol{\theta}}_{1}^{T} \\
\underline{\boldsymbol{\theta}}_{2}^{T} \\
\underline{\boldsymbol{\theta}}_{3}^{T} \\
\underline{\boldsymbol{\theta}}_{4}^{T}
\end{array}\right] .
\end{gathered}
$$

The details of the robust Wiener model output with the upper bound $\bar{y}$ and the lower bound $y$ is shown in Fig. 4, with the validation. It is obvious that the measured output variable $y$ is in the band defined by robust Wiener model. This approach enables the modeling of nonlinear dynamical systems with uncertainties. It can also be easily used to model the family of nonlinear time-invariant systems with uncertain physical parameters. It is, therefore, useful in all applications where those problems arise, especially in the case of fault detection or in the case of robust control design.

\section{CONCLUSION}

The problem of interval fuzzy model identification and its use in the case of the robust Wiener model is addressed in this correspondence. The proposed approach enables the modeling of nonlinear timeinvariant systems with uncertainties. The resulting model defines the lower and upper model outputs that define the confidence interval of the observed data. The interval fuzzy model is used to approximate the static nonlinearity in the case of the robust Wiener model. This approach to the modeling of nonlinear dynamical systems has the potential for use in the areas of robust control and fault detection.

\section{REFERENCES}

[1] H. Tanaka, S. Uejima, and K. Asai, "Linear regression analysis with fuzzy model," IEEE Trans. Syst., Man, Cybern., vol. SMC-12, no. 6, pp. 903-907, 1982.

[2] H. Tanaka and J. Watada, "Possibilistic linear systems and their applications to the linear regression model," Fuzzy Sets Syst., vol. 27, pp. 275-289, 1988.

[3] G. Peters, "Fuzzy linear regression with fuzzy intervals," Fuzzy Sets Syst., vol. 63, pp. 45-55, 1994.

[4] Y. S. Chen, "Outliers detection and confidence interval modification in fuzzy regression," Fuzzy Sets Syst., vol. 119, no. 2, pp. 259-272, 2001.

[5] B. Turksen, “Type I and type II fuzzy system modeling," Fuzzy Sets Syst., vol. 106, pp. 11-34, 1999.

[6] N. Karnik and J. Mendel, "Operation on type-2 fuzzy sets," Fuzzy Sets Syst., vol. 122, no. 2, pp. 327-348, 2001.

[7] Q. Liang and J. Mendel, "Interval type-2 fuzzy logic systems: Theory and design," IEEE Trans. Fuzzy Syst., vol. 8, no. 5, pp. 535-550, Oct. 2000.

[8] B. Kosko, "Fuzzy systems as universal approximators," IEEE Trans. Comput., vol. 43, no. 11, pp. 1329-1333, Nov. 1994.

[9] H. G. C. Ying, "Necessary conditions for some typical fuzzy systems as universal approximators," Automatica, vol. 33, pp. 1333-1338, 1997.

[10] L.-X. Wang and J. M. Mendel, "Fuzzy basis functions, universal approximation, and orthogonal least-squares learning," IEEE Trans. Neural Netw., vol. 3, no. 5, pp. 807-814, Sep. 1992.

[11] R. R. Goldberg, Methods of Real Analysis. New York: Wiley, 1976.

[12] C.-H. Lin, "Siso nonlinear system identification using a fuzzy-neural hybrid system," Int. J. Neural Syst., vol. 8, no. 3, pp. 325-337, 1997.

[13] P. Julian, M. Jordan, and A. Desages, "Canonical piecewise linear approximation of smooh functions," IEEE Trans. Circ. Syst., vol. 45, no. 5, pp. 567-571, May 1998.

[14] S. Gerkšič, D. Juričič, S. Strmčnik, and D. Matko, "Wiener model based nonlinear predictive control," Int. J. Syst. Sci., vol. 31, no. 2, pp. 189-202, 2000

[15] J. C. Bezdek, C. Coray, R. Gunderson, and J. Watson, "Detection and characterization of cluster substructure, I. Linear structure: Fuzzy c-lines," SIAM J. Appl. Math., vol. 40, no. 2, pp. 339-357, 1981.

[16] R. J. Hathaway, C. J. Bezdek, and Y. K. Hu, "Generalized fuzzy c-means clustering strategies using Lp norm distances," IEEE Trans. Fuzzy Syst., vol. 8, no. 5, pp. 576-582, Oct. 2000.

[17] L. Ljung, System Identification. Englewood Cliffs, NJ: Prentice-Hall, 1987. 